\title{
A Procedure for the Selection of Single Sampling Plans by Variables Based on Pareto Distribution
}

\author{
Geetha Sathya Narayanan $^{1}$ and Vijayaraghavan Rajarathinam ${ }^{2}$ \\ ${ }^{1}$ Department of Statistics, PSG College of Arts and Science, Coimbatore, Tamil Nadu 641 014, India \\ ${ }^{2}$ Department of Statistics, Bharathiar University, Coimbatore, Tamil Nadu 641 046, India
}

Correspondence should be addressed to Vijayaraghavan Rajarathinam; rvijayrn@yahoo.com

Received 16 December 2012; Revised 13 August 2013; Accepted 20 August 2013

Academic Editor: Yi-Hung Chen

Copyright ( 2013 G. S. Narayanan and V. Rajarathinam. This is an open access article distributed under the Creative Commons Attribution License, which permits unrestricted use, distribution, and reproduction in any medium, provided the original work is properly cited.

\begin{abstract}
Product control in statistical quality control is the methodology that deals with procedures for taking decisions about one or more lots of finished products manufactured by production processes. Sampling inspection by variables is one of the major classifications of product control and comprises procedures for deciding about the disposition of a lot of individual units based on sample measurements of units on a quality characteristic under study. These procedures are defined under the assumption that the quality characteristic is measurable on a continuous scale, and the functional form of the probability distribution must be known. Inspection procedures which have been developed under the implicit assumption that the quality characteristic is distributed as normal with the related properties are found in the literature of product control. The assumption of normality may not be realized often in practice, and it becomes indispensable to investigate the properties of sampling plans based on nonnormal distributions. In this paper, a single sampling plan by variables is formulated and evaluated when the quality characteristic is assumed to be distributed according to a Pareto distribution. A procedure is developed for determining the parameters of the plan for specified requirements to ensure protection to both the producer and consumer.
\end{abstract}

\section{Introduction}

Sampling inspection is an activity for taking decisions on one or more lots of finished products which have been submitted for inspection. The decision of either acceptance or rejection of the lots is usually taken by adopting suitable sampling inspection procedures called sampling plans. Sampling plans are generally categorized into two types, namely, lot-by-lot sampling by attributes and lot-by-lot sampling by variables. In lot-by-lot inspection by attributes, one or more samples of items are drawn from a given lot of manufactured items; each item in the sample(s) is classified as conforming or nonconforming, and the decision of acceptance or rejection of the lot is made based on a specific rule. In lot-bylot inspection by variables, one or more samples of items are drawn from a given lot; the measurement of a quality characteristic in each sampled item is recorded, and the decision of acceptance or rejection of the lot is made as a function of such measurements. The theory of inspection by variables is applicable when the quality characteristic of sampled items is measurable on a continuous scale and the functional form of the probability distribution is assumed to be known. A variables sampling is advantageous in the sense that it generates more information from each item inspected, requires small sample, and provides same protection when compared to attributes sampling. (See Bowker and Goode [1] and Montgomery [2]).

On the basis of the implicit assumption that the quality characteristic is distributed according to normal with mean $\mu$ and standard deviation $\sigma$, the concept of variables sampling inspection has been studied by many researchers. Some of the early works on variables sampling inspection are seen in Lieberman and Resnikoff [3], Schilling [4], Owen [5, 6], and Hamaker [7]. Studies relating to sampling plans when the assumption of normality of the quality characteristic fails or the functional form of the underlying distribution deviates from normal or the form of the distribution is not known are also found in the literature of acceptance sampling. 
Duncan [8], Srivastava [9], Das and Mitra [10], Owen [11], Takagi [12], Guenther [13, 14], Zimmer and Burr [15], Aminzadeh [16], Suresh and Ramanathan [17], Vijayaraghavan and Geetha [18-20], and Geetha and Vijayaraghavan [21, 22] are few references which deal with variables inspection using nonnormal distributions.

When the quality characteristic $X$ follows normal distribution with mean $\mu$ and standard deviation $\sigma$, the problem of designing single sampling plans by variables is addressed with ease. In the industrial situations, quite often, the assumption of normality may not be valid or the quality characteristic may be distributed according to non-normal distributions. In such cases, the selection of variable sampling plans becomes complicated. However, the literature of acceptance sampling traces several works which deal with the designing of variables plans when the quality characteristic follows a probability distribution other than normal. Owen [11] presented a detailed survey on various works related to variable sampling plans with emphasis on nonnormality.

In this paper a study on single sampling plans by variables is formulated under the assumption that the quality characteristics have a Pareto distribution. A procedure for determining the parameters of the proposed plan for specified requirements in terms of producer's and consumer's protection is also developed.

\section{Single Sampling Inspection Plans by Variables}

A single sampling inspection plan by variables is defined under the following assumptions.

(a) The quality characteristic, denoted by $X$, is measurable on a continuous scale and has a known form of probability distribution, represented by $F_{X}(x ; \mu, \sigma)$, which is the distribution function of $X$ with mean $\mu$ and variance $\sigma^{2}$.

(b) Each individual unit in a submitted lot has a onesided specification say, lower specification, $L$ or upper specification $U$. If, for a unit, $X>U$ (or $X<L$ ), the unit is classified as a nonconforming unit.

The operating procedure of a variable sampling plan is as follows.

Step 1. Draw a random sample of $n$ units from a lot and observe the measurements $x_{1}, x_{2}, \ldots, x_{n}$ of the quality characteristic $X$.

Step 2. When $\sigma$ is known, accept the lot if $\bar{x}+k \sigma \leq U$ (or $\bar{x}-k \sigma \geq L)$; otherwise, reject the lot where $\bar{x}$ is the sample mean. When $\sigma$ is unknown, accept the lot if $\bar{x}+k s \leq U$ (or $\bar{x}-k s \geq L)$, where $s^{2}=(1 /(n-1)) \sum_{i: 1}^{n}\left(x_{i}-\bar{x}\right)^{2}$ is an unbiased estimate of $\sigma^{2}$.

Thus, a single sampling plan by variables is designated by two parameters, namely, the sample size, $n$, and the acceptability constant, $k$. When these parameters are known, the plan could be implemented. The explicit expressions for $n$ and $k$ can be derived by specifying two points on the operating characteristic curve of the plan, namely, $\left(p_{1}, 1-\right.$ $\alpha)$ and $\left(p_{2}, \beta\right)$, where $p_{1}$ and $p_{2}$ are termed as acceptable quality level (AQL), and the limiting quality level (LQL) associated with the producer's risk $\alpha$ and the consumer's risk $\beta$, respectively. A sampling plan by variables is termed as a known $\sigma$ or unknown $\sigma$ plan according to whether $\sigma$ is known or unknown.

\section{Operating Characteristic Function}

An important measure of the performance of a variables sampling plan is its operating characteristic function, which is a function of the proportion $p$ of non-conforming units called incoming lot quality, and it provides the probability, $P_{a}(p)$ of acceptance of a lot. The plot of $P_{a}(p)$ against $p$ results in a curve called the operating characteristic (OC) curve. For a given upper specification limit $U$ when $\sigma$ is known, $p$ and $P_{a}(p)$ are defined by

$$
\begin{gathered}
p=P(X>U \mid \mu), \\
P_{a}(p)=P(\bar{x}+k \sigma \leq U \mid \mu) .
\end{gathered}
$$

AQL and LQL, using (1), are defined by

$$
\begin{aligned}
& \mathrm{AQL}=p_{1}=P\left(X>U \mid \mu_{1}\right), \\
& \mathrm{LQL}=p_{2}=P\left(X>U \mid \mu_{2}\right),
\end{aligned}
$$

where $\mu_{1}$ and $\mu_{2}$ are the means of the underlying distribution which results in AQL and LQL, respectively.

Assume that $X$ is modeled by the Pareto distribution with parameters $a$ and $b$. Then, its probability density function and the cumulative distribution function are, respectively, given by

$$
\begin{gathered}
f(x)=\frac{a b^{a}}{x^{a+1}}, \quad x>b, a<0, \\
F(x)=1-\left(\frac{b}{x}\right)^{a} .
\end{gathered}
$$

The mean and the variance of the underlying distribution are given by $\mu=a b /(a-1)$ for $a>1$ and $\sigma^{2}=a b^{2} /(a-$ $1)^{2}(a-2)$ for $a>2$, respectively. Further, the measures of skewness and kurtosis, which are the measures for identifying the asymmetric nature of the distribution, are expressed as $\alpha_{3}=(2(1+a) /(a-3)) \sqrt{(a-2) / a}$ for $a>3$ and $\alpha_{4}=3(a-$ $2)\left(3 a^{3}+a+2\right) / a(a-3)(a-4)$, respectively. From (1) and (3), the lot quality levels $p, \mathrm{AQL}$, and LQL using standard Pareto distribution are, respectively, defined by

$$
\begin{gathered}
p=P\left(T>K_{p}^{*}\right), \\
\mathrm{AQL}=p_{1}=P\left(T>K_{p_{1}}^{*}\right), \\
\mathrm{LQL}=p_{2}=P\left(T>K_{p_{2}}^{*}\right),
\end{gathered}
$$

where $T=(X-\mu) / \sigma, K_{p}^{*}=(U-\mu) / \sigma, K_{p_{1}}^{*}=\left(U-\mu_{1}\right) / \sigma$, and $K_{p_{2}}^{*}=\left(U-\mu_{2}\right) / \sigma$. 
The producer's risk $\alpha$ and the consumer's risk $\beta$ corresponding to AQL and LQL are, respectively, defined from (2) as

$$
\begin{gathered}
\alpha=P\left(\bar{x}+k \sigma>U \mid \mu=\mu_{1}\right), \\
1-\beta=P\left(\bar{x}+k \sigma>U \mid \mu=\mu_{2}\right) .
\end{gathered}
$$

When $\sigma$ is unknown, the estimate $s$ is used in the decision criterion and hence in the evaluation of $\alpha$ and $\beta$.

\section{Designing Single Sampling Plans by Variables}

In the industrial practice the unknown standard deviation variables plans are more realistic than the known standard deviation variables plans. If the distribution is non-normal, then the designing of unknown $\sigma$ plans is much complicated. Takagi [12] attempted to provide solution to such problems and proposed a methodology for determining the parameters of variables sampling plans under the conditions of nonnormal populations by introducing an expansion factor in terms of the measures of skewness and kurtosis. The procedures of designing known and unknown standard deviation sampling plans giving protection to the producer and consumer are discussed in [18-22] under the assumption that the quality characteristics under study follow gamma, Laplace, Rayleigh and logistic distributions when the measures of skewness and/or kurtosis are specified.

The methodology proposed in [12] using the expansion factor will, now, be discussed for an unknown sigma plan by variables under the assumption of Pareto distribution for the quality characteristic.

In the case of unknown sigma plan, the determination of $n$ and $k$ is usually based on the sampling distribution of $\bar{x}+k s$ or $\bar{x}-k s$. When the underlying distribution is normal, $\bar{x}$ and $s$ are independently distributed as normal and hence $\bar{x}+k s$ and $\bar{x}-k s$ are also normally distributed. By this property, explicit forms of $n$ and $k$ could be derived for determining $n$ and $k$. A linear combination of independently distributed variables tends to be normally distributed even when the individual variables depart from normality (see [15]). Further, when the underlying distribution is non-normal, according to [12], the asymptotic distributions of $\bar{x}+k s$ and $\bar{x}-k s$ are normal with the means, respectively, given by $\mu_{y}=\mu+k \sigma$ and $\mu_{y}=\mu-k \sigma$, and with the common variance expressed by

$$
\sigma_{Y}^{2}=\frac{\sigma^{2}}{n}\left[1+\frac{k^{2}}{4}\left(\alpha_{4}-1\right) \pm k \alpha_{3}\right]
$$

where $\alpha_{3}$ and $\alpha_{4}$ represent the measures of skewness and kurtosis of the underlying distribution.

It is known that $K_{p}^{*}=(U-\mu) / \sigma$. Thus, for specified $p_{1}$ and $p_{2}$,

$$
\begin{aligned}
& \mu_{1}=U-K_{p_{1}}^{*} \sigma, \\
& \mu_{2}=U-K_{p_{2}}^{*} \sigma .
\end{aligned}
$$

The operating characteristic function $P_{a}(p)$ for an unknown standard deviation plan is expressed as

$$
P_{a}(p)=P_{r}\left[\bar{x}+k_{U} s \leq U \mid p\right]=P_{r}(Y \leq U \mid p) .
$$

Assume that $p_{1}$ and $p_{2}$ are specified with the risks $\alpha$ of rejecting the lot and $\beta$ of accepting the lot. Then, corresponding to $\alpha$ and $\beta$, the normal deviates $K_{\alpha}$ and $K_{\beta}$ are, respectively, defined by

$$
\begin{aligned}
K_{\alpha} & =\frac{U-\left(\mu_{1}+k \sigma\right)}{\sqrt{\left(\sigma^{2} / n\right)\left[1+\left(k^{2} / 4\right)\left(\alpha_{4}-1\right)+k \alpha_{3}\right]}} \\
& =\frac{U-\left(\mu_{1}+k_{U} \sigma\right)}{\sigma \sqrt{e_{U} / n_{U}}}, \\
-K_{\beta} & =\frac{U-\left(\mu_{2}+k \sigma\right)}{\sqrt{\left(\sigma^{2} / n\right)\left[1+\left(k^{2} / 4\right)\left(\alpha_{4}-1\right)+k \alpha_{3}\right]}} \\
& =\frac{U-\left(\mu_{2}+k_{U} \sigma\right)}{\sigma \sqrt{e_{U} / n_{U}}} .
\end{aligned}
$$

On substituting the expressions (12) for $\mu_{1}$ and $\mu_{2}$, (14) would result in the following:

$$
\begin{aligned}
& K_{p_{1}}^{*}=k_{U}+K_{\alpha} \sqrt{\frac{e_{U}}{n_{U}}}, \\
& K_{p_{2}}^{*}=k_{U}-K_{\beta} \sqrt{\frac{e_{U}}{n_{U}}} .
\end{aligned}
$$

The mathematical expressions for $n$ and $k$ of an unknown standard deviation plan for specified requirements in terms of the points $\left(p_{1}, 1-\alpha\right)$ and $\left(p_{2}, \beta\right)$ on the OC curve such that $P_{a}\left(p_{1}\right)=1-\alpha$ and $P_{a}\left(p_{2}\right)=\beta$, when $U$ is specified, are derived from (15) as

$$
\begin{aligned}
& n_{U}=e_{U}\left[\frac{K_{\alpha}+K_{\beta}}{K_{p_{1}}^{*}-K_{p_{2}}^{*}}\right]^{2}, \\
& k_{U}=\frac{K_{\alpha} K_{p_{2}}^{*}+K_{\beta} K_{p_{1}}^{*}}{K_{\alpha}+K_{\beta}},
\end{aligned}
$$

where $K_{\alpha}$ and $K_{\beta}$ are the standardized deviates exceeded with the probabilities $\alpha$ and $\beta$, respectively, and $e_{U}$ is an expansion factor, which is a function of $\alpha_{3}$ and $\alpha_{4}$, expressed as $e_{U}=$ $1+\left(k_{U}^{2} / 4\right)\left(\alpha_{4}-1\right)+k_{U} \alpha_{3}$. The expansion factor is used to obtain the known standard deviation plans with parameters $n^{\prime}$ and $k^{\prime}$ by using the relations $n_{U}^{\prime}=n_{U} / e_{U}$ and $k_{U}^{\prime}=k_{U}$. In a similar way, when $L$ is specified, the expressions for $n$ and $k$ are given by

$$
\begin{aligned}
& n_{L}=e_{L}\left[\frac{K_{\alpha}+K_{\beta}}{K_{1-p_{1}}^{*}-K_{1-p_{2}}^{*}}\right]^{2}, \\
& k_{L}=-\frac{K_{\alpha} K_{1-p_{2}}^{*}+K_{\beta} K_{1-p_{1}}^{*}}{K_{\alpha}+K_{\beta}},
\end{aligned}
$$


where $e_{L}$ is expressed by $e_{L}=1+\left(k_{L}^{2} / 4\right)\left(\alpha_{4}-1\right)-k_{L} \alpha_{3}$, using which the known standard deviation plan with parameters $n^{\prime}$ and $k^{\prime}$ could be derived as $n_{L}^{\prime}=n_{L} / e_{L}$ and $k_{L}^{\prime}=k_{L}$.

\section{Numerical Example}

Suppose that a set of measurements yields $\alpha_{3}=2.2671$ and $\alpha_{4}=11.3626$. It is desired to determine a variables sampling plan giving protection to the producer and the consumer in terms of $\left(p_{1}=0.01, \alpha=0.05\right)$ and $\left(p_{2}=\right.$ $0.06, \beta=0.10)$. For the given requirements, the value of $a$ is calculated from the expression of $\alpha_{3}$ of Pareto distribution as 25. Corresponding to $p_{1}$ and $p_{2}$, the values of $K_{p_{1}}^{*}$ and $K_{p_{2}}^{*}$ are determined, respectively, from (7) and (8) as 3.6970 and 1.7828. The normal deviates $K_{\alpha}$ and $K_{\beta}$ are obtained as 1.64485 and 1.28155 by satisfying (9) and (10) for specified values of $\alpha$ and $\beta$. Substituting these values in (16) and (17), the parameters of the desired plan are determined as $n=$ 57.8256 which when rounded becomes 58 and $k=2.6211$. The value of $e_{U}$ is obtained as 24.7401 . Thus, the parameters of a known standard deviation plan are computed as $n_{U}^{\prime}=$ $n_{U} / e_{U}=2.3373 \approx 3$ and $k_{U}^{\prime}=2.6211$.

\section{A Note on Deriving the Plans Based on Exact Pareto Distribution}

It is known that Pareto distribution is a very heavy-tailed distribution for which the $r$ th moment is finite when the shape parameter $a$ is greater than $r$. Thus, large observations can be expected in samples from this distribution. Further, the distribution function and the maximum likelihood estimators of the parameters can be written in closed form. As the distribution is not in general symmetric and the results given in the previous sections are based only on the assumption of a symmetric distribution, it would be appropriate if one could derive variables sampling plans using the exact Pareto distribution. Whereas it would be reasonable to assume that the mean is finite, no assumption about finite moments has to be made if one uses the median in lieu of mean. It is to be noted that the median could be used as a measure of location when a distribution is skewed (say, for instance, a Pareto distribution) or when end values are not known or when one requires reduced importance to be attached to outliers. When sample median is used in the operating procedure of a sampling plan for making a decision about the lot, the explicit expressions for the plan parameters are not plausible to derive. In such a case, the procedure for finding the parameters based on exact Pareto distribution will be based on the sampling distribution of sample median and will be much complicated.

\section{Conclusion}

Sampling inspection by variables is the methodology used for deciding about the disposition of a lot based on sample measurements of units on a quality characteristic under study. Such a procedure is defined under the assumption that the quality characteristic is measurable on a continuous scale and the functional form of the probability distribution is known. Though sampling plans by variables under the implicit assumption that the quality characteristic is distributed as normal have been developed with the related properties, it should be realized that the assumption of normality is seldom hold in practice. Hence, it is inevitable to develop sampling plans by variables when the underlying quality characteristics follow non-normal distributions, which may be skewed or symmetric. In this paper a single sampling plan by variables is formulated and evaluated when the quality characteristic is distributed according to a Pareto distribution. The approximation of normal distribution for a linear combination of independent variables even when the individual variables depart from normality is effectively used in developing the procedure for determining the parameters of the plan when requirements for producer's and the consumer's protection are specified.

\section{Acknowledgments}

The authors are grateful to the Editor/Editorial Board Member of the journal and anonymous referees who have made significant suggestions for the improvements in the content of the paper. They also acknowledge University Grants Commission, New Delhi, India, for providing financial support to carry out this work.

\section{References}

[1] A. H. Bowker and H. P. Goode, Sampling Inspection by Variables, McGraw-Hill, New York, NY, USA, 1952.

[2] D. C. Montgomery, Introduction to Statistical Quality Control, John Wiley \& Sons, New York, NY, USA, 2004.

[3] G. J. Lieberman and G. J. Resnikoff, "Sampling plans for inspection by variables," Journal of the American Statistical Association, vol. 50, pp. 457-516, 1955.

[4] E. G. Schilling, Acceptance Sampling in Quality Control, Marcel Dekker, New York, NY, USA, 1982.

[5] D. B. Owen, "One-sided variables sampling plans," Industrial Quality Control, vol. 22, pp. 450-4456, 1966.

[6] D. B. Owen, "Variables sampling plans based on the normal distribution," Technometrics, vol. 9, pp. 417-423, 1967.

[7] H. C. Hamaker, "Acceptance sampling for percent defective by variables and by attributes," Journal of Quality Technology, vol. 11, no. 3, pp. 139-148, 1979.

[8] A. J. Duncan, Quality Control and Industrial Statistics, Edited by: R. D. Irwin, Homewood, Chicago, Ill, USA, 1986.

[9] A. B. L. Srivastava, "Variables sampling inspection for nonnormal samples," Journal of Science and Engineering Research, vol. 5, pp. 145-152, 1961.

[10] N. G. Das and S. K. Mitra, "The effect of non-normality on plans for sampling inspection by variables," Sankhya-A, vol. 26, pp. 169-176, 1964.

[11] D. B. Owen, "Summary of recent work on variables acceptance sampling with emphasis on non-normality," Technometrics, vol. 11, no. 4, pp. 631-637, 1969.

[12] K. Takagi, "On designing unknown-sigma sampling plans based on a wide class of non-normal distributions," Technometrics, vol. 14, no. 3, pp. 669-678, 1972. 
[13] W. C. Guenther, "Variables sampling plans for the poisson and the binomial," Statistica Neerlandica, vol. 26, pp. 113-120, 1972.

[14] W. C. Guenther, "LQL like plans for sampling by variables," Journal of Quality Technology, vol. 17, pp. 155-157, 1985.

[15] W. J. Zimmer and I. W. Burr, "Variables sampling plans based on non-normal populations," Industrial Quality Control, vol. 21, pp. 18-26, 1963.

[16] M. S. Aminzadeh, "Inverse-gaussian acceptance sampling plans by variables," Communications in Statistics-Theory and Methods, vol. 25, no. 5, pp. 923-935, 1996.

[17] R. P. Suresh and T. V. Ramanathan, "Acceptance sampling plans by variables for a class of symmetric distributions," Communications in Statistics-Simulation and Computation, vol. 26, no. 4, pp. 1379-1391, 1997.

[18] R. Vijayaraghavan and S. Geetha, "Evaluation of single sampling plans by variables using single parameter gamma distribution," in Proceedings of the National Conference on Quality Improvement Concepts and their Implementation in Higher Educational Institutions, Amrita University, Coimbatore, India, December 2009.

[19] R. Vijayaraghavan and S. Geetha, "Procedure for selection of single sampling plans variables based on gamma distribution," in UGC National Level Staff Seminar on Applied Statistics, Sri Sarada College for Women, Salem, India, December 2009.

[20] R. Vijayaraghavan and S. Geetha, "Procedure for selection of single sampling plans variables based on Laplace distribution," in Recent Trends in Statistical Research, pp. 209-218, Publication Division, Manonmaniam Sundaranar University, 2010.

[21] S. Geetha and R. Vijayaraghavan, "Evaluation of single sampling plan by variables based on rayleigh distribution," in Proceedings of the National Conference on Statistics for Twenty First Century (NCSTC) \& Annual Conference of Kerala Statistical Association, University of Kerala, Trivandrum, India, March 2011.

[22] S. Geetha and R. Vijayaraghavan, "Selection of single sampling plans by variables based on logistic distribution," in Proceedings of the UGC Sponsored National Conference on Recent Trends in Statistical and Computer Applications, Manonmaniam Sundaranar University, Tirunelveli, India, March 2011. 

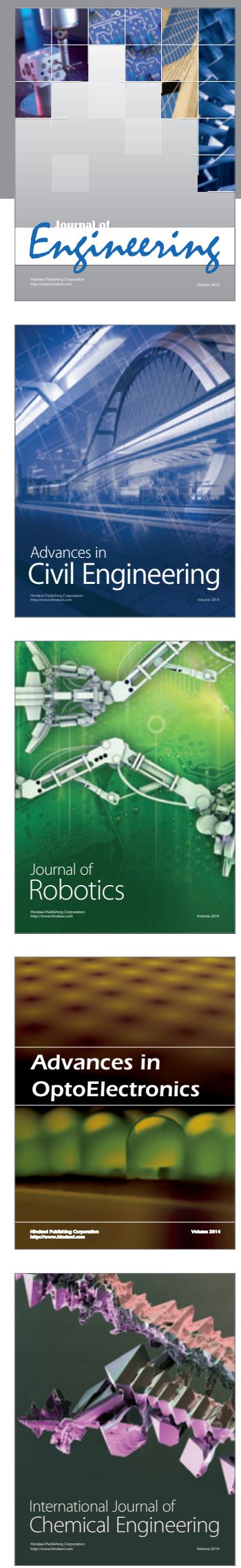

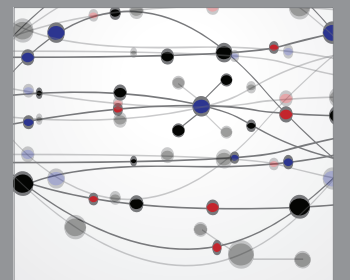

The Scientific World Journal
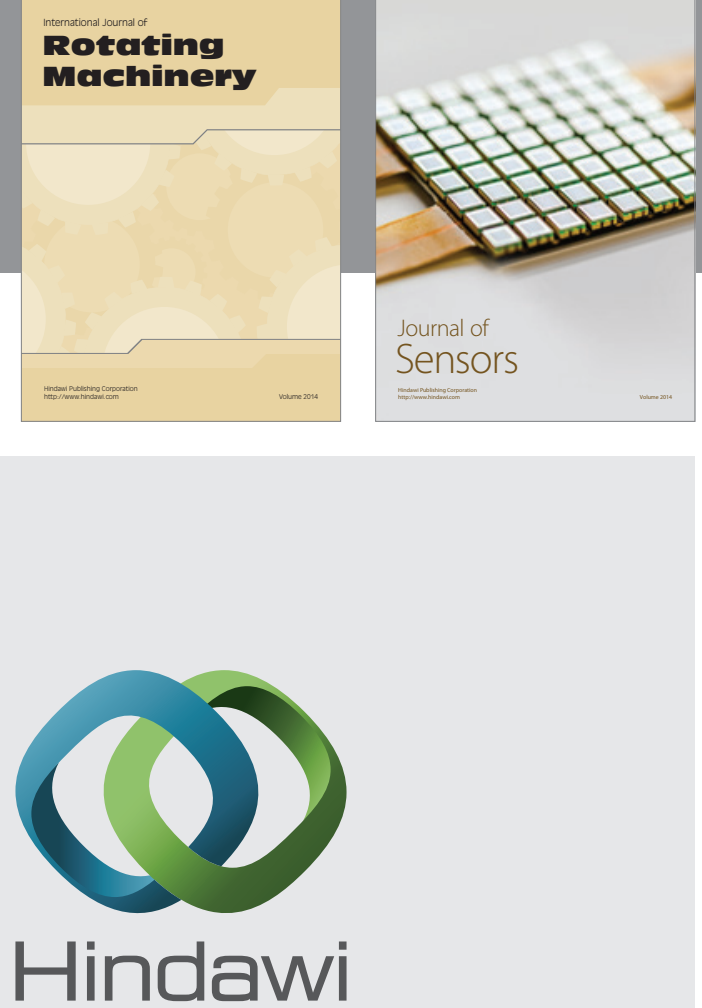

Submit your manuscripts at http://www.hindawi.com
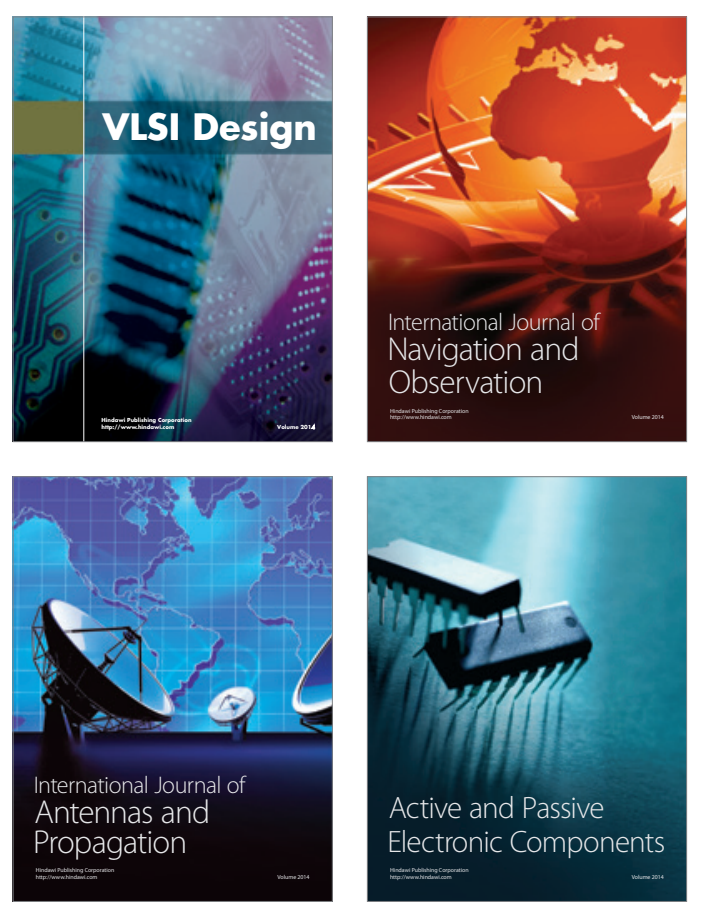
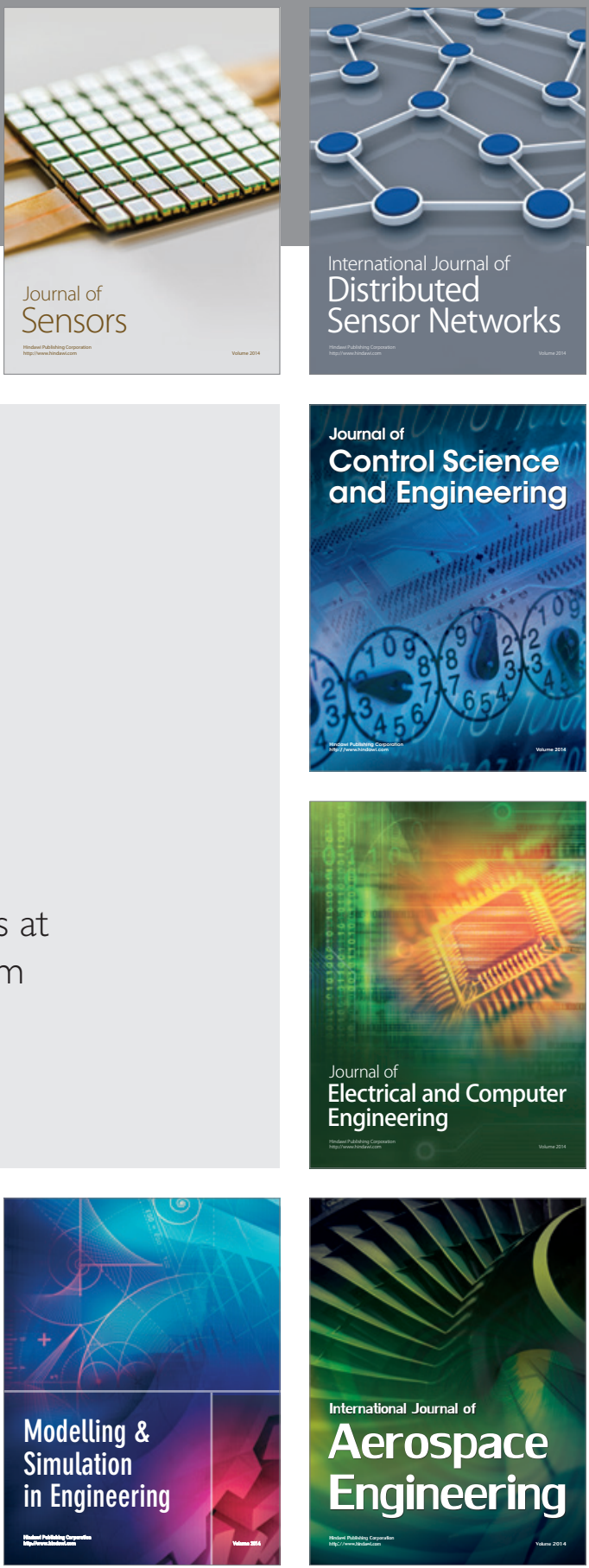

Journal of

Control Science

and Engineering
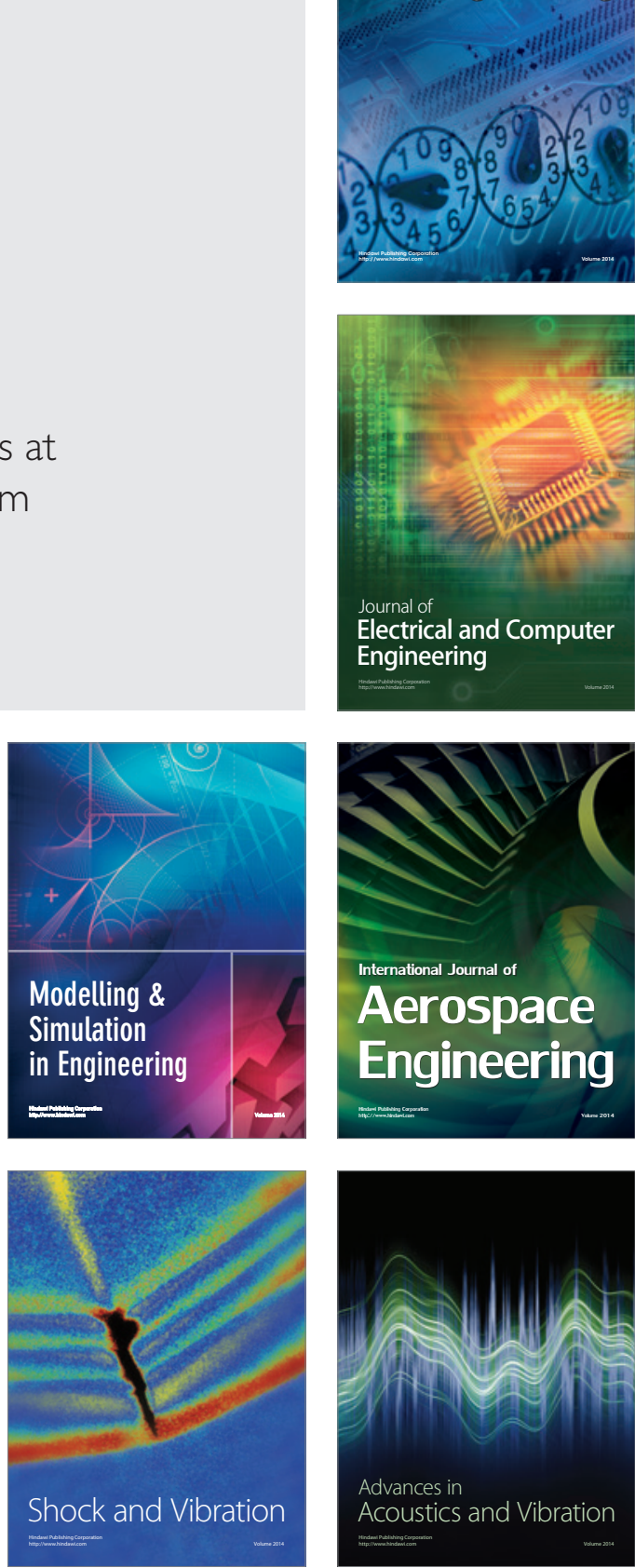